\title{
Validação cruzada entre o percentual de gordura mensurado pela absortometria radiológica de dupla energia e a equação de Deurenberg em idosas
}

\author{
Cross validation between the percentage of fat measured by dual energy x-ray absorptiometry and \\ the equation of Deurenberg in elderly women

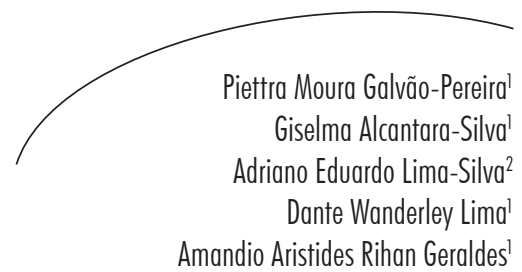

Resumo

Objetivo: Avaliar a validade cruzada da equação de Deurenberg et al. Métodos: Estudo transversal descritivo, no qual 25 mulheres (59,04 $\pm 7,2$ anos) foram selecionadas aleatoriamente de uma população de 186 mulheres com idade a partir de 50 anos, envolvidas em programas de atividade física de lazer em Maceió-AL. O método de referência utilizado foi a absortometria radiológica de dupla energia (DXA). O percentual de gordura corporal foi estimado pela equação de Deurenberg et al., que utiliza como variáveis independentes: índice de massa corporal, idade e sexo. $\mathrm{O}$ teste $t$ foi utilizado para comparar as médias; o coeficiente de correlação intraclasse (CCI) para determinar a correlação e gráficos de Bland \& Altman para avaliar a concordância. Resultados: Nenhuma diferença significativa foi observada entre os dois métodos $(\mathrm{p}=0,358)$, além de um elevado CCI $(0,84 ; \mathrm{p}<0,05)$. O erro constante indicou que o percentual de gordura encontrado pela equação subestima ligeiramente $(1,1 \%)$ o valor obtido pela DXA. Um erro padrão da estimativa de 6,1\% foi observado e a distribuição dos resíduos individuais dos gráficos de Bland \& Altman mostrou limites de concordância (95\%), variando entre 12,88 e -10,67\%. Conclusão: A equação proposta por Deurenberg et al. é válida para estimar o percentual de gordura em mulheres com características semelhantes à amostra do presente estudo.

\section{Abstract}

Objective: To evaluate the validity of cross-equation Deurenberg et al. Methods: Crosssectional study in which 25 women (59.04 \pm 7.2 years) were randomly selected from a population of 186 women aged from 50 years, involved in programs of leisure physical activity in Maceió city, state of Alagoas, Brazil. The reference method used was the X-ray absorptiometry (DXA). The percentage of body fat was estimated by the equation

Palavras-chave:

Antropometria. Obesidade. Envelhecimento. Composição Corporal.

\footnotetext{
Programa de Pós-graduação em Nutrição, Faculdade de Nutrição. Universidade Federal de Alagoas. Maceió, AL, Brasil.

2 Grupo de Pesquisa em Ciências do Esporte, Faculdade de Nutrição. Universidade Federal de Alagoas. Maceió, AL, Brasil.
} 
of Deurenberg et al., using as independent variables: body mass index, age and sex. The $t$ test was used to compare averages, the intraclass correlation coefficient (ICC) to determine the correlation and Bland \& Altman to assess agreement. Results: No significant difference was observed between the two methods $(\mathrm{p}=0.358)$, and a high ICC $(0.84, \mathrm{p}<0.05)$. The constant error indicated that the percentage of fat found by equation underestimates slightly $(1.1 \%)$ the value obtained by DXA. A standard error of estimate of $6.1 \%$ was observed and the distribution of individual residues of Bland \& Altman showed limits of agreement (95\%), ranging between 12.88 and $-10.67 \%$. Conclusion: The equation proposed by Deurenberg et al. is valid to estimate the percentage of fat in women with characteristics similar to the study sample.

\section{Key words:}

Anthropometry. Obesity.

Aging. Body Composition.

\section{INTRODUÇÃO}

As associações verificadas entre $\mathrm{o}$ índice de massa corporal (IMC) com a mortalidade ${ }^{1-3}$ e o aumento das taxas de risco para doenças, como infarto do miocárdio, ${ }^{4}$ acidente vascular cerebral,${ }^{5}$ diabetes, ${ }^{6}$ obesidade ${ }^{7}$ e diversos tipos de cânceres, ${ }^{7,8}$ além da adiposidade corporal, ${ }^{3,9}$ fornecem a base lógica para uso do IMC na predição da obesidade. Ademais, o IMC oferece vantagens evidentes e indiscutíveis, visto que, além da facilidade de obtenção e rápido acesso, as medidas necessárias para seu cálculo apresentam elevada reprodutibilidade e exigem um treinamento simples e equipamentos pouco onerosos, ${ }^{9,10} \mathrm{o}$ que pode auxiliar $\mathrm{O}$ desenvolvimento de políticas de atenção à saúde da pessoa idosa.

O IMC tem sido o recurso mais utilizado para avaliar os riscos de saúde relacionados ao sobrepeso e à obesidade, ${ }^{10-12}$ mas seu uso como medida única para a avaliação dos riscos relacionados à adiposidade corporal - ou percentual de gordura $(\% \mathrm{G})$ - não é consensual. Oreupoulos et al. ${ }^{13}$ e Rezende et al. ${ }^{14}$ defendem que o IMC apresenta baixa sensibilidade para identificar e diferenciar os indivíduos com sobrepeso e/ou obesidade.

Diante do acelerado envelhecimento populacional e do crescente aumento das taxas de obesidade e comorbidades associadas verificadas na população idosa, ${ }^{7,15}$ nomeadamente entre as mulheres, ${ }^{15}$ a disponibilidade de recursos destinados à predição da gordura corporal (GC) que utilizam variáveis de fácil acesso, como é o caso do IMC, além de desejável, permite menor desconforto e maior adesão dos sujeitos ao processo de avaliação da composição corporal. Nessa direção, Deurenberg et al. ${ }^{16}$ desenvolveram uma equação que, utilizando o IMC, a idade e o sexo como variáveis independentes, foi capaz de estimar a GC em idosos.

Apesar de ter sido validada em idosos, a equação desenvolvida no estudo de Deurenberg et al. ${ }^{16}$ utilizou-se de uma amostra de idosos europeus, mais precisamente holandeses. Portanto, diante das possíveis diferenças morfológicas, relacionadas à genética, às influências ambientais e socioculturais, estilo de vida e condições de saúde e funcionalidade dos sujeitos ${ }^{17}$ antes de utilizar generalizadamente uma equação de regressão, é necessário verificar sua validade cruzada em outras amostras. ${ }^{17-19}$

Portanto, utilizando-se como método de referência a absortometria radiológica de dupla energia (DXA), o objetivo deste estudo foi avaliar a validade cruzada da equação de Deurenberg et al., ${ }^{16}$ a fim de verificar a adequabilidade de seu uso numa amostra composta por mulheres com idades a partir de 50 anos.

\section{MATERIAL E MÉTODOS}

População e amostra

A população-alvo do presente estudo foi composta por 186 mulheres que, engajadas em programas de atividades físicas de lazer ofertadas por instituições de terceira idade do município de Maceió, aceitaram participar como voluntárias 
da pesquisa. Para compor a amostra deste estudo descritivo, ${ }^{20}$ ou levantamento transversal, foram selecionadas por sorteio simples, dentre a população citada, 65 mulheres que atenderam aos critérios de inclusão: serem fisicamente ativas, funcionalmente independentes e com idades acima de 50 anos.

Seriam excluídos do estudo os sujeitos que: 1) reportassem possuir quaisquer doenças que pudessem afetar o sistema músculo-esquelético (exemplo: neuropatias, doença de Cushing, doença pulmonar obstrutiva crônica, câncer ativo ou tratamento recente para câncer); 2) utilizassem medicamentos capazes de alterar a composição corporal (exemplo: corticoides, andrógenos ou drogas antiandrógenas e antipsicóticos); 3) tivessem sofrido infarto do miocárdio recentemente; 4) possuíssem massa corporal superior a $100 \mathrm{~kg}$ (devido à capacidade de atenuação da DXA); 5) tivessem o perímetro do quadril maior que $65 \mathrm{~cm}$ (largura da mesa da DXA) e 6) os que não tiveram todos os dados da avaliação da composição corporal e da entrevista coletados.

Para verificação dos critérios de inclusão e exclusão, os sujeitos foram entrevistados com auxílio de um questionário geral, desenvolvido para o estudo, pelo questionário de avaliação funcional multidimensional (OARS) e pela aferição das medidas antropométricas. Após a avaliação dos critérios de inclusão e exclusão, 25 mulheres com idades entre 50 e 73 anos (59,04 \pm 7,2 anos) foram submetidas às avaliações.

Este estudo foi conduzido de acordo com as normas da Resolução n. 196/96 do Conselho Nacional de Saúde sobre pesquisa envolvendo seres humanos, tendo sido aprovado pelo Comitê de Ética em Pesquisa da Universidade Federal de Alagoas (UFAL), processo no: 020.487/2008-53. Todos os sujeitos que aceitaram ser voluntários do estudo assinaram o Termo de Consentimento Livre e Esclarecido.

Procedimentos

Antes da realização das medidas antropométricas e da DXA, foram feitas as recomendações necessárias como descrito por Rezende et al. ${ }^{14}$ Ao chegar ao laboratório, na manhã agendada para os exames, foram realizadas as medidas antropométricas e, em seguida, o exame da DXA nas voluntárias.

\section{Medidas antropométricas}

Todas as participantes foram medidas com roupas leves, respeitando-se os protocolos recomendados por Lohman et al. ${ }^{21}$ A massa corporal foi expressa em quilogramas (com sensibilidade de $0,1 \mathrm{~kg}$ ) e realizada a medição em uma balança mecânica (Filizola ${ }^{\circledR}$, São Paulo, Brasil); a estatura, expressa em metros (com sensibilidade de $0,05 \mathrm{~m}$ ), foi mensurada por meio de um estadiômetro portátil de parede (Seca ${ }^{\circledR}$, Baystate Scale \& Systems, USA).

Para o cálculo do índice de massa corporal (IMC), utilizou-se a razão entre a massa corporal e o quadrado da estatura, expresso em $\mathrm{kg} / \mathrm{m}^{2}$.

\section{Estimativa do percentual de gordura por meio da equação}

Para a predição da GC (\%GIMC), utilizouse a equação proposta por Deurenberg et al., ${ }^{16}$ onde: $\%$ GIMC $=1,20$ (IMC) + 0,23 (IDADE) 10,8 (SEXO); a idade é aferida por meio dos anos completos, sendo o sexo expresso pelo número " 0 ", visto que todas eram mulheres.

\section{Medida da gordura corporal por meio da DXA}

Para a medida da gordura corporal, utilizou-se a DXA (\%GDXA), método que vem sendo utilizado como critério em estudos semelhantes. ${ }^{16,22}$ Foi utilizado um aparelho do fabricante Lunar modelo DPX-IQ (Lunar Radiation, Madison, WI; software versão 4.7, modo estendido). Para garantir as qualidades psicométricas, o aparelho era diariamente averiguado quanto à calibração, com phantom 
cedido pelo fabricante, de acordo com os padrões descritos no manual do equipamento.

Para garantir a reprodutibilidade, as medidas obtidas pela DXA e necessárias para a avaliação da composição corporal dos sujeitos, foram realizadas no mesmo horário e pelo mesmo técnico. Os resultados e os laudos relacionados aos mesmos foram elaborados e avaliados por médico radiologista. Dentre as possíveis variáveis fornecidas pela DXA - tecido mole magro, tecido mole gordo, conteúdo mineral ósseo, densidade óssea e $\% \mathrm{G}$, do corpo inteiro e de partes deste -, neste estudo utilizou-se a medida do $\% \mathrm{G}$ total (\%GDXA).

\section{Tratamento estatístico}

A normalidade e a homogeneidade da amostra foram avaliadas pelos testes ShapiroWilk e Levene, respectivamente. A estatística descritiva média, amplitude (maior e menor valor) e desvio-padrão foram utilizados para organizar e apresentar os dados. O \%GIMC e \%GDXA foram comparados pelo teste $t$ pareado. A correlação entre as duas técnicas foi realizada por meio do coeficiente de correlação intraclasse (CCI). A plotagem proposta por Bland \& Altman ${ }^{23,24}$ foi utilizada para analisar a distribuição dos resíduos individuais entre os métodos para um intervalo de confiança de $95 \%$. Todos os cálculos estatísticos foram realizados com auxílio do pacote estatístico Statistical Package for the Social Science, versão SPSS ${ }^{\circledR} 12.0$ (Chicago, IL, USA), considerando-se, para todos os testes estatísticos, um nível de significância de p<0,05.

\section{RESULTADOS}

A apresentação dos resultados da idade, medidas antropométricas, bem como do \%G obtido por meio das duas técnicas, pode ser observada na tabela 1.

Tabela 1 - Características dos sujeitos $(n=25)$ e resultados dos percentuais de gordura obtidos pela equação de Deurenberg e absortometria radiológica de dupla energia. Maceió-AL, 2010.

\begin{tabular}{lcc}
\hline \multicolumn{1}{c}{ Variáveis } & $\mathrm{m} \pm \mathrm{dp}$ & $\begin{array}{c}\text { Amplitude } \\
(\mathrm{mv}-\mathrm{MV})\end{array}$ \\
\hline Idade (anos) & $59,04 \pm 7,2$ & $50-73$ \\
Estatura (m) & $1,57 \pm 0$ & $1,43-1,74$ \\
MC $(\mathrm{kg})$ & $62,8 \pm 7,2$ & $62,8-84$ \\
$\mathrm{IMC}\left(\mathrm{kg} / \mathrm{m}^{2}\right)$ & $25,5 \pm 5,2$ & $18,7-40,4$ \\
$\%$ GDXA & $39,92 \pm 9$ & $19-57$ \\
$\%$ GIMC & $38,81 \pm 7$ & $29,5-56,2$ \\
\hline
\end{tabular}

$\mathrm{m}=$ média aritmética; $\mathrm{dp}=$ desvio-padrão da média; $\mathrm{mv}=$ menor valor; $\mathrm{MV}=$ maior valor; $\mathrm{MC}=$ massa corporal; $\mathrm{IMC}=$ índice de massa corporal; $\% \mathrm{GDXA}=$ percentual de gordura obtido por meio da absortometria; $\% \mathrm{GIMC}=$ resultado do percentual de gordura calculado por meio da equação de Deurenberg.

Com relação aos critérios de validação (tabela 2), não houve diferença estatisticamente significativa entre as médias dos \%GC obtidos pelos dois métodos $(\mathrm{p}=0,358)$. Além disso, o
CCI indicou forte relação entre os métodos. Com relação ao erro constante (EC), o \%GIMC subestimou o valor do \%GDXA em 1,1\%. 
Tabela 2 - Validação cruzada entre a equação de Deurenberg et al. e absortometria radiológica de dupla energia ( $\mathrm{n}=25)$. Maceió-AL, 2010.

\begin{tabular}{cccccc}
\hline Variável & $t$ & $\mathrm{p}$ & $\mathrm{CCI}$ & $\mathrm{EC}$ & $\mathrm{EPE}$ \\
\hline$\%$ GIMC vs $\%$ GDXA & $-0,92$ & 0,368 & 0,84 & $1,1 \%$ & $6,1 \%$ \\
\hline
\end{tabular}

$t=$ resultado da comparação entre as médias das duas técnicas ( $t$ Student pareado); CCI= coeficiente de correlação intraclasse; EC $=$ erro constante (diferença entre as médias das duas técnicas); EPE = erro padrão da estimativa.

Os resultados da concordância entre os resultados obtidos pelos dois métodos, testados com auxílio da plotagem gráfica de Bland \& Altman, ${ }^{23}$ podem ser verificados na figura 1.

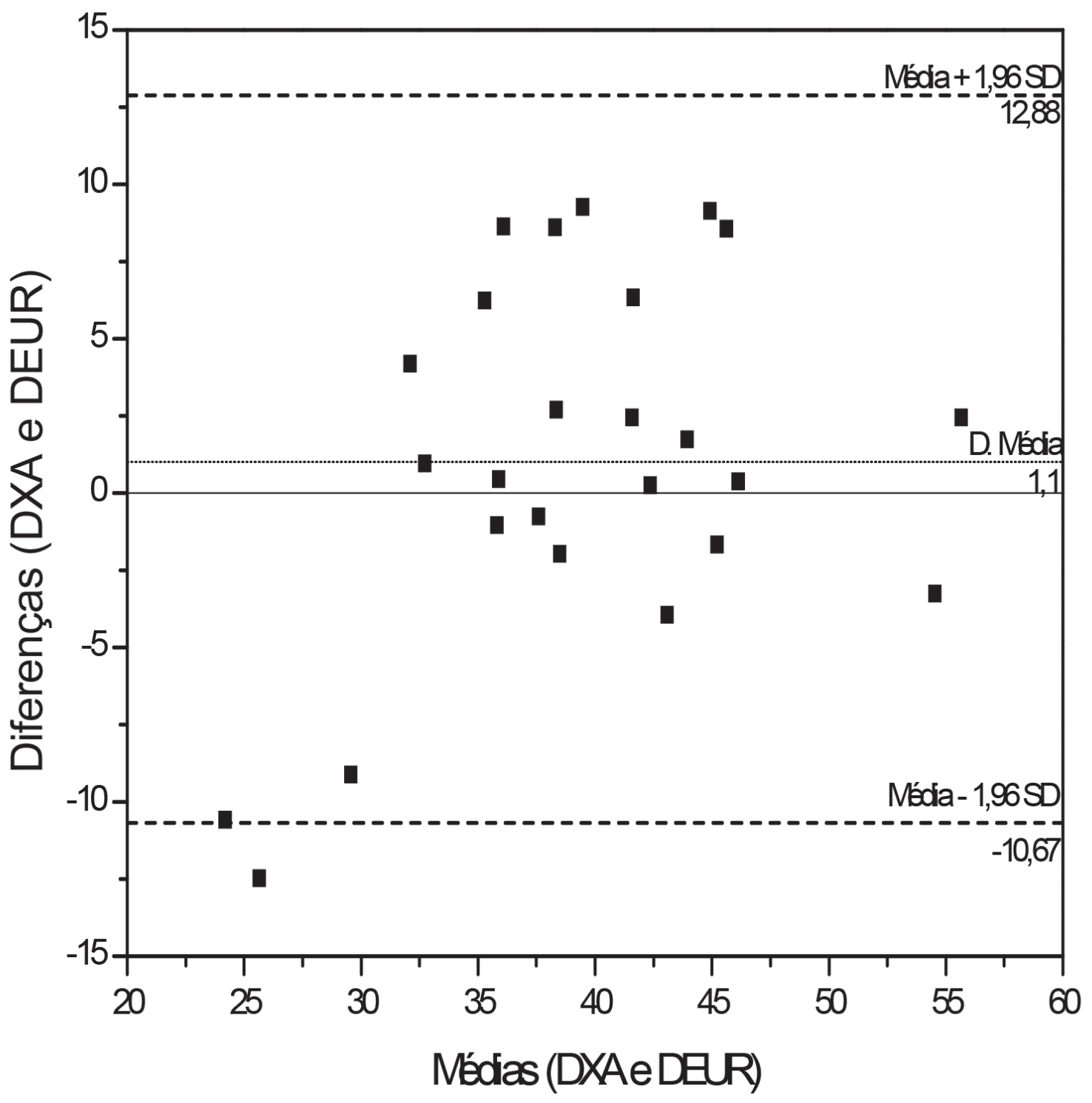

Figura 1 - Plotagem de Bland \& Altman para os limites de concordância entre os valores de gordura corporal estimados pela equação de Deurenberg e DXA. Maceió-AL, 2010. 


\section{DISCUSSÃO}

O estudo destinou-se a estabelecer a validação cruzada da equação proposta por Deurenberg et al. ${ }^{16}$ para avaliação da gordura corporal de mulheres com idades a partir de 50 anos (59,04 $\pm 7,2$ anos). Considerando que esse recurso é de fácil aplicabilidade e baixo custo, a validação de seu uso fornece uma ferramenta importante para avaliação da composição corporal de idosos.

Considerando-se o \%GDXA como referência (tabela 1) e a classificação proposta por Lohman, ${ }^{25}$ que propõe que sejam classificadas como obesas as mulheres que apresentem $\% \mathrm{G}$ iguais ou maiores que $32 \%$, a amostra foi classificada como obesa, quando avaliada pelas duas técnicas - \%GIMC e \%GDXA. Por outro lado, quando considerado o valor médio do IMC, classificado de acordo com os pontos de corte propostos especificamente para idosos, ${ }^{26}$ a amostra seria classificada como eutrófica.

Aparentemente conflitantes, esses resultados podem ser explicados pelas alterações morfológicas ocorridas durante o processo de envelhecimento, visto que, com a senescência, além de haver aumento na quantidade de gordura corporal, esta é redistribuída, passando a se concentrar nas regiões abdominal e intramuscular..$^{15}$ Adicionalmente, verifica-se diminuição da densidade mineral óssea ${ }^{27}$ e da quantidade de massa muscular esquelética, ${ }^{2}$ especialmente entre as mulheres pós-menopausadas, ${ }^{2,27}$ levando a uma baixa sensibilidade do IMC para detectar a gordura corporal.

Equações antropométricas de maior precisão e praticidade para utilização em pesquisas de campo poderiam eliminar vieses decorrentes de mudanças morfológicas próprias do envelhecimento. No presente estudo, quando realizada a comparação entre os resultados dos $\% \mathrm{G}$ verificados pelas duas técnicas (\%GIMC e \%GDXA) por meio do teste $t$ pareado, não foram verificadas diferenças estatisticamente significantes $(\mathrm{p}=0,368)$.

Guedes \& Rechenchoski, ${ }^{28}$ utilizando como amostra sujeitos adultos de ambos os sexos (174 moças e 257 rapazes) e idades entre 18 e 30 anos, com o objetivo de verificar a validação de quatro diferentes equações antropométricas (utilizando dobras cutâneas) com a equação de Deurenberg et al., ${ }^{16}$ também não observaram diferenças estatísticas entre as técnicas. Entretanto, comparações com tal estudo são problemáticas, devido à utilização de uma amostra com diferentes características etárias, morfológicas, e níveis de atividades físicas das observadas no presente estudo. Além disso, naquele estudo, os padrões de referência utilizados foram equações preditivas que utilizam as dobras cutâneas (DC). Quanto aos CCI, estes variaram entre 0,46 e 0,61, a depender da equação utilizada. Os baixos CCI podem ter sido observados devido ao maior erro inter e intra-avaliador, derivado da utilização da técnica de DC em sujeitos obesos e idosos. ${ }^{22,29}$

Na mesma direção, no Sul do Brasil, Rech et al., ${ }^{22} \mathrm{em}$ amostra composta por 180 idosos de ambos os sexos (120 mulheres e 60 homens), com idades entre 60 e 81 anos, verificaram a validade cruzada de sete equações antropométricas para a estimativa do $\% \mathrm{G}$, dentre as quais a equação de Deurenberg et al., ${ }^{16}$ e observaram elevado CCI ( 0,74 para homens e 0,75 para mulheres) entre os métodos. Entretanto, diferentemente do presente estudo, Rech et al. ${ }^{22}$ observaram diferença significativa entre as médias do \%GDXA e \%GIMC. A diferença estatística entre os métodos observada por Rech etal. ${ }^{22}$ e o fato de a significância estatística entre os métodos ter sido observada no presente estudo pode ter sido referida devido à média de IMC observada nas duas amostras. A população de idosos sulistas do Brasil apresentou maior média de IMC $\left(27,4 \pm 3,9 \mathrm{~kg} / \mathrm{m}^{2}\right)$ do que a amostra de idosas nordestinas que compôs o presente estudo $\left(25,5 \pm 5,2 \mathrm{~kg} / \mathrm{m}^{2}\right)$. Dessa forma, um IMC em torno de $25 \mathrm{~kg} / \mathrm{m}^{2}$ pode indicar maior validade do \%GIMC, fato demonstrado também pelo propositor da técnica, ${ }^{16}$ quando observou menores erros de estimativa $(0,2 \%) \mathrm{em}$ sujeitos com IMC entre 20 e $25 \mathrm{~kg} / \mathrm{m}^{2}$ e maiores erros $(0,6 \%)$ de estimativa em sujeitos com IMC $\geq 30 \mathrm{~kg} / \mathrm{m}^{2}$.

Em se tratando da correlação observada entre as duas técnicas, no presente estudo, observou- 
se CCI igual a 0,84 - na realidade maior que o verificado pelos propositores originais da técnica $^{16}(\mathrm{r}=0,79)$. Em seu estudo, Deurenberg et $a 1 .{ }^{16}$ utilizaram uma amostra composta por sujeitos de ambos os sexos, com amplitude de idade entre sete e 83 anos, e a hidrodensitometria foi utilizada como medida de referência. Deurenberg et al. ${ }^{9}$ realizaram um segundo estudo de validação, com sujeitos de ambos os sexos (234 mulheres e 182 homens), com idades entre 18 e 70 anos, com o \%GC avaliado desta vez pela DXA e pela hidrodensitometria, e encontraram um coeficiente de correlação igual a 0,78 . O valor do CCI mais elevado do presente estudo pode ter sido encontrado devido às características homogêneas em relação à idade, níveis de atividade física e funcionalidade observados nas idosas que compuseram a amostra.

No que se refere ao erro padrão de estimativa (EPE), interpretado como o desvio-padrão de todos os erros ou resíduos produzidos por uma equação de predição (\%GIMC), no presente estudo foi observado o valor de 6,1\%. Segundo Lohman, ${ }^{25}$ esse valor deve ser de até 3,5\%, quando se pretende estimar uma variável.

Diante da normalidade observada na distribuição dos dados, pode-se então afirmar que $68 \%$ dos dados estimados pelo $\%$ GIMC variaram em $\pm 6,1 \%$, o que pode causar erros de classificação individual dos sujeitos quanto à quantidade de gordura corporal. Entretanto, considerando a aplicabilidade da técnica, a utilização de variáveis de fácil acesso como massa corporal, estatura e, sobretudo, pelo fato de a idade ser considerada variável independente do \%G, a utilização da equação de Deurenberg et al. ${ }^{16}$ é uma boa ferramenta para utilização em grandes amostras. Nos estudos de Deurenberg et al., ${ }^{9,16}$ por exemplo, os EPEs verificados foram, respectivamente, $4,1 \%$ e 4,8\%. No estudo de Rech et al., ${ }^{22}$ o EPE encontrado para homens foi de $3,6 \%$; para mulheres, o valor não foi apresentado, visto ter diferido da média do método critério.

Em se tratando do erro constante (EC) obtido por meio da diferença entre as médias dos percentuais de gordura verificados, no estudo de Rech et al. ${ }^{22}$ observou-se, nos sujeitos do sexo masculino, um EC de $0,5 \%$ (IC $=95 \%$, limites de concordância $=-7,5 \%$ a $8,5 \%$ ). $\mathrm{Na}$ presente pesquisa, o EC foi de 1,1\%, o que demonstra pequena diferença entre as médias dos resultados dos percentuais de gordura obtidos pelos dois métodos.

$\mathrm{Na}$ análise de Bland \& Altman, ${ }^{23}$ os limites dos intervalos de confiança (média $\pm 1,96$ desvios-padrão) estabeleceram-se entre 12,88\% e $-10,67 \%$, demonstrando certa variabilidade quanto à concordância entre os valores estimados por ambos os métodos e apontando para a possibilidade de má classificação dos sujeitos quanto à quantidade de gordura corporal. Os sujeitos podem ser classificados como obesos, mas podem não sê-lo, bem como podem ser classificados como eutróficos, sendo obesos.

Diante do reduzido número de estudos relacionados ao tema, e das limitações apresentadas pelo presente estudo, nomeadamente no que se refere ao pequeno tamanho da amostra e ao fato de todos os sujeitos serem fisicamente ativos e do sexo feminino, sugere-se, para um entendimento mais abrangente sobre a utilização generalizada da equação preditiva de Deurenberg et al., ${ }^{16}$ a realização de novas pesquisas com metodologia semelhante, mas utilizando amostras maiores compostas por sujeitos de ambos os sexos e de diferentes níveis de aptidão física.

\section{CONCLUSÃO}

Com base nestes resultados, pode-se concluir que a equação proposta por Deurenberg et al., apesar de apresentar elevado erro padrão de estimativa, é válida para estimar o percentual de gordura em mulheres com idades a partir dos 50 anos e características semelhantes às da amostra do presente estudo, especialmente em estudos populacionais. $\mathrm{O}$ teste $t$ não demonstrou diferença estatística entre os métodos, e foram verificados elevado coeficiente de correlação $(0,84)$ e baixo erro constante $(1,1 \%)$. No entanto, por apresentar elevado erro padrão de estimativa, deve-se ter cautela quanto a seu uso em classificações individuais. 


\section{REFERÊNCIAS}

1. Flegal KM, Graubard BI. Estimates of excess deaths associated with body mass index and other anthropometric variables. Am J Clin Nutr 2009;89(4):1213-9.

2. Janssen I, Mark AE. Elevated body mass index and mortality risk in the elderly. Obes Rev 2007;8(1):41-59.

3. Whitlock G, Lewington S, Sherliker P, Clarke R, Emberson J, Halsey J, et al; Prospective Studies Collaboration. Body-mass index and cause-specific mortality in 900000 adults: collaborative analyses of 57 prospective studies. Lancet 2009;373(9669):1083-96.

4. de Koning L, Merchant AT, Pogue J, Anand SS. Waist circumference and waist-to-hip ratio as predictors of cardiovascular events: meta-regression analysis of prospective studies. Eur Heart J 2007;28(7):850-6.

5. Ma SH, Park BY, Yang JJ, Jung EJ, Yeo Y, Whang $\mathrm{Y}$, et al. Interaction of body mass index and diabetes as modifiers of cardiovascular mortality in a cohort study. J Prev Med Public Health 2012;45(6):394-401.

6. Hartemink N, Boshuizen HC, Nagelkerke NJ, Jacobs $\mathrm{MA}$, van Houwelingen HC. Combining risk estimates from observational studies with different exposure cutpoints: a meta-analysis on body mass index and diabetes type 2. Am J Epidemiol 2006;163(11):1042-52.

7. Lenz M, Richter T, Mühlhauser I. The morbidity and mortality associated with overweight and obesity in adulthood: a systematic review. Dtsch Arztebl Int 2009;106(40):641-8.

8. Dai Z, Xu YC, Niu L. Obesity and colorectal cancer risk: a meta-analysis of cohort studies. World J Gastroenterol 2007;13(31):4199-206.

9. Deurenberg P, Andreoli A, Borg P, KukkonenHarjula K, de Lorenzo A, van Marken Linchtenbelt WD, et al. The validity of predicted body fat percentage from body mass index and from impedance in samples of five European populations. Eur J Clin Nutr 2001;55(11):973-9.

10. Herman KM, Hopman WM, Vandenkerkhof EG, Rosenberg MW. Physical activity, body mass index, and health-related quality of life in Canadian adults. Med Sci Sports Exerc 2012;44(4):625-36.

11. Renzaho A, Wooden M, Houng B. Associations between body mass index and health-related quality of life among Australian adults. Qual Life Res 2010;19(4):515-20.

12. Kortt MA, Dollery B. Association between body mass index and health-related quality of life among an Australian sample. Clin Ther 2011;33(10):1466-74.
13. Oreopoulos A, Padwal R, Kalantar-Zadeh K, Fonarow GC, Norris CM, McAlister FA. Body mass index and mortality in heart failure: a meta-analysis. Am Heart J 2008;156(1):13-22.

14. Rezende FAC, Rosado LEFPL, Franceschinni SCC, Rosado GP, Ribeiro RCL. Aplicabilidade do índice de massa corporal na avaliação da gordura corporal. Rev Bras Med Esporte 2010;16(2):90-4.

15. Zamboni M, Mazzali G, ZoicoE, Harris TB, Meigs JB, Di Francesco V, et al. Health consequences of obesity in the elderly: a review of four unresolved questions. Int J Obes (Lond) 2005;29(9):1011-29.

16. Deurenberg P, Weststrate JA, Seidell JC. Body mass index as a measure of body fatness: ageand sex-specific prediction formulas. Br J Nutr 1991;65(2):105-14.

17. Leahy S, O'Neill C, Sohun R, Toomey C, Jakeman P. Generalised equations for the prediction of percentage body fat by anthropometry in adult men and women aged 18181 years. Br J Nutr 2012;29:1-8.

18. Loftin M, Nichols J, Going S, Sothern M, Schmitz $\mathrm{KH}$, Ring $\mathrm{K}$, et al. Comparison of the validity of anthropometric and bioelectric impedance equations to assess body composition in adolescent girls. Int J Body Compos Res 2007;5(1):1-8.

19. Fonseca PHS, Marins JCB, Silva AT. Validação de equações antropométricas que estimam a densidade corporal em atletas profissionais de futebol. Rev Bras Med Esporte 2007;13(3):153-6.

20. Thomas JR, Nelson JK. Métodos de pesquisa em atividade física e saúde. 3. ed. São Paulo: Artmed Editora; 2002.

21. Lohman TG, Roche A, Martorell R. Anthropometric stadization reference manual. Champaign, Illinois: Human Kinetics Publishers; 1988.

22. Rech CR, Lima LRA, Cordeiro BA, Petroski EL, Vasconcelos FAG. Validade de equações antropométricas para estimativa da gordura corporal em idosos do sul do Brasil. Rev Bras Cineatropom Desempenho Hum 2010;12(1):1-7.

23. Bland JM, Altman DG. Statistical methods for assessing agreement between two methods of clinical measurement. Lancet 1986;1(8476):307-10.

24. Martelli Filho JA, Maltagliati LA, Trevisan F, Alcântara GCTL. Novo método estatístico para análise da reprodutibilidade. Rev Dent Press Ortodon Ortopedi Facial 2005;10(5):122-9. 
25. Lohman TG. Advances in body composition assessments. Champaign, Illinois: Human Kinetics Publishers; 1992.

26. Lipschitz DA. Screening for nutritional status in the elderly. Prim Care 1994;21(1):55-67.

27. Waugh EJ, Lam MA, Hawker GA, McGowan J, Papaioannou A, Cheung AM, et al; Perimenopause BMD Guidelines Subcommittee of Osteoporosis Canada. Risk factors for low bone mass in healthy
40-60 year old women: a systematic review of the literature. Osteoporos Int 2009;20(1):1-21.

28. Guedes DP, Rechenchosky L. Comparação da gordura corporal predita por métodos antropométricos: índice de massa corporal e espessuras de dobras cutâneas. Rev Bras Cineantropom Desempenho Hum 2008;10(1):1-7.

29. Heyward VH. ASEP methods recommendation: body composition assessment. JEPonline 2001;4(4):1-12.

Recebido: 04/12/2012

Revisado: 07/7/2013

Aprovado: 30/7/2013 